\title{
Intracerebral hemorrhage (ICH) evaluation with a novel magnetic induction sensor: a preliminary study using the Chinese head model
}

\author{
Ziyi Zhang ${ }^{\mathrm{a}, \mathrm{b}, *}$, Peiguo Liu ${ }^{\mathrm{b}}$, Dongming Zhou ${ }^{\mathrm{b}}$, Liang Zhang ${ }^{\mathrm{a}, \mathrm{b}}$ and Hengdong Lei ${ }^{\mathrm{a}, \mathrm{b}}$ \\ ${ }^{a}$ College of Science, National University of Defense Technology, Changsha, Hunan 410073, China \\ ${ }^{\mathrm{b}}$ State Key Laboratory of Complex Electromagnetic Environmental Effects on Electronics \& \\ Information System, National University of Defense Technology, Changsha, Hunan 410073, China
}

\begin{abstract}
Biomedical magnetic induction measurement is a promising method for the detection of intracerebral hemorrhage (ICH), especially in China. Aiming at overcoming the problem of low sensitivity, a magnetic induction sensor is chosen to replace the conventional sensors. It uses a two-arm Archimedean spiral coil as the exciter and a circular coil as the receiver. In order to carry out high-fidelity simulations, the Chinese head model with real anatomical structure is introduced into this novel sensor for the first time. Simulations have been carried out upon early stage ICH measurements. By calculating the state sensitivity and time sensitivity of the perturbation phase of two types of sensors using the electromagnetic software, we conclude that the primary signal received can be largely reduced using the novel sensor, which could effectively increase the time and state sensitivity simultaneously.
\end{abstract}

Keywords: Intracerebral hemorrhage (ICH), magnetic induction measurement, sensor sensitivity, two-arm archimedean spiral coil (TAASC), Chinese head model

\section{Introduction}

Intracerebral hemorrhage (ICH) is a common and devastating stroke with high disability and death rates all over the world. As for China, the situation is even worse. It has been reported that the incidence of ICH in China is higher than that in the western countries, and a huge economic burden is imposed on the Chinese government every year $[1,2]$. Although there are numerous non-invasive imaging techniques (e.g., CT and MRI) which have been applied in clinical examination and can be used to determine the size and location of the intracranial lesion accurately, yet some drawbacks still exist.

- Limited availability and expensive diagnostic test of devices in developing countries including China;

\footnotetext{
${ }^{*}$ Corresponding author: Ziyi Zhang, Room 605, Electromagnetic Environment Laboratory Building, College of Electronic Science and Engineering, National University of Defense Technology, 109 Deya Road, Kaifu District, Changsha, Hunan 410073, China. Tel.: +86 073184576219 15; Fax: +86 0731 84576219; E-mail: ziyizhang@nudt.edu.cn.
}

0959-2989/14/\$27.50 @ 2014 - IOS Press and the authors. 
- Inconvenience of carrying and operating due to the devices' bulkiness and complexity;

- Unable to real-time and long-term monitoring;

- Insensitivity to early stage diseases.

Biomedical magnetic induction measurement has been attracting a lot of attention in ICH diagnosis recently [3-6]. As the passive electromagnetic properties of human tissues are affected by their physiological states, bleeding in the brain will cause local increase of electrical conductivity [7]. Based on this principle, magnetic induction tomography (MIT) is able to map the electrical conductivity of the brain to detect ICH, which belongs to functional imaging methods. Although MIT has low resolution when compared with morphological imaging techniques, it is both low-cost and fast, which allows it to be utilized as a supplementary tool for early stage ICH evaluation [8]. Nevertheless, one major difficulty with MIT is its low sensitivity. To simplify the structure and ease the analysis, a circular coil is used as the exciter for the conventional sensor. The disadvantage of the circular excitation coil is that the magnetic field it emits (primary signal) around the receiver is so strong that the magnetic field induced from the perturbation object (secondary signal) is submerged [9].

In order to improve the proportion of the secondary signal in the total signal sensed in the receiver, a novel magnetic induction sensor is designed. It employs a two-arm Archimedean spiral coil (TAASC) as the exciter and a circular coil as the receiver, with the asymmetric configuration of TAASC successfully reducing the strength of the primary signal. In this paper, the Chinese head model with real anatomical structure is firstly introduced into this novel sensor. Besides, early stage ICH measurements are simulated using the commercial electromagnetic computation software CST MW STUDIO (CST AG) for both types of sensors to show a comparison.

\section{Methods}

\subsection{Principle of ICH measurement with magnetic induction method}

Figure 1(a) illustrates the principle of ICH measurement using magnetic induction method. The sensor is a single channel system with an excitation coil and a receiver coil. A sinusoidal current, with frequency $f$ and magnitude $I$, is fed into the excitation coil, producing the primary magnetic field which can be quantified as a primary voltage $V_{0}$ by the receiver coil. If a hemorrhage occurs in the brain, the primary magnetic field will induce eddy current, radiating the secondary magnetic field which can be measured by the receiver coil as a secondary voltage $\Delta V$. The perturbation phase $\Delta \varphi$ of the received signal is of interest as it contains the information of electrical conductivity in the hemorrhage spot. It has been confirmed that the perturbation phase shows the linearity relation with the change of electrical conductivity of lesion [9]:

$$
\operatorname{Im}\left(\frac{\Delta V}{V_{0}}\right) \approx \Delta \varphi \propto \omega \Delta \sigma
$$

where $\omega$ is the angular frequency. The value of $\Delta \sigma$ can be obtained by measuring $\Delta \varphi$, which then determines whether there is a hemorrhage. 


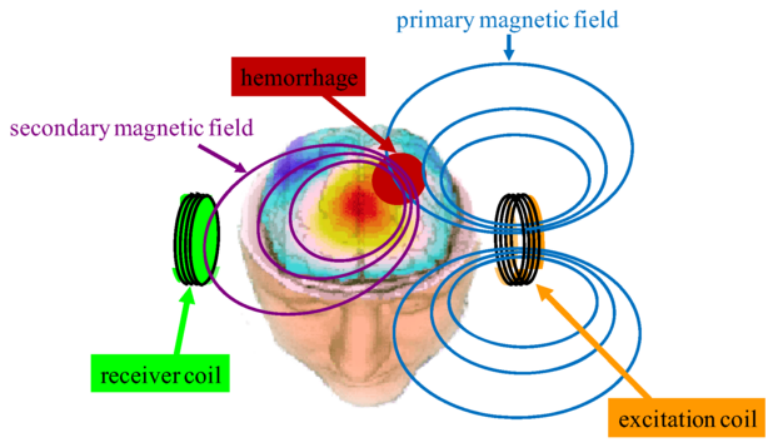

(a)

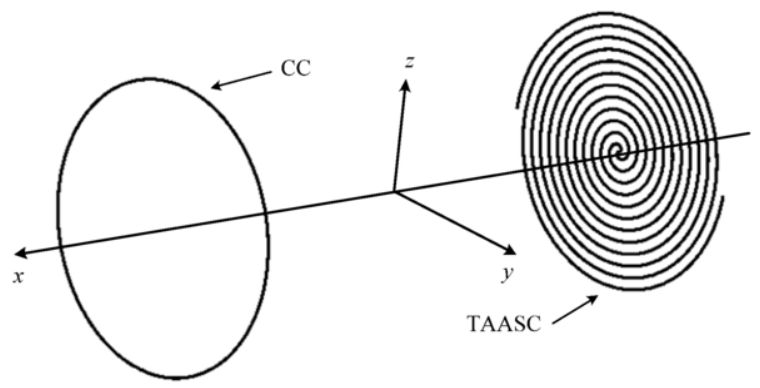

(b)

Fig. 1. (a) Sketch map for the principle of ICH measurement using magnetic induction method [7]; (b) Novel magnetic induction sensor.

\subsection{Magnetic induction sensor}

The novel magnetic induction sensor is shown in Figure 1(b), where the exciter is a two-arm Archimedean spiral coil (TAASC), and the receiver is a circular coil (CC). The TAASC is constructed by two opposite Archimedean spirals connected in the center, and both the Archimedean spirals have turns of six. The CC has one turn, and is placed coaxially with respect to the TAASC. The TAASC and CC have the same radius $r$, and the same distance $d$ to the $y-z$ plane.

As the dimension of the sensor is much smaller than the wavelength of the excitation current, the $x$ component of the magnetic field transmitted by the TAASC can be calculated using the static electromagnetic field theory

$$
\begin{aligned}
& B_{x}=\frac{\mu_{0} r I}{48 \pi^{2}}\left[\int _ { 0 } ^ { 1 2 \pi } \left(\frac{\left(\left(\cos \varphi_{1}-\varphi_{1} \sin \varphi_{1}\right)\left(z-(r / 12 \pi) \varphi_{1} \sin \varphi_{1}\right)\right)}{\left(\left(y-(r / 12 \pi) \varphi_{1} \cos \varphi_{1}\right)^{2}+\left(z-(r / 12 \pi) \varphi_{1} \sin \varphi_{1}\right)^{2}+(x+d)^{2}\right)^{3 / 2}}-\right.\right. \\
& \left.\frac{\left(\left(\sin \varphi_{1}+\varphi_{1} \cos \varphi_{1}\right)\left(y-(r / 12 \pi) \varphi_{1} \cos \varphi_{1}\right)\right)}{\left(\left(y-(r / 12 \pi) \varphi_{1} \cos \varphi_{1}\right)^{2}+\left(z-(r / 12 \pi) \varphi_{1} \sin \varphi_{1}\right)^{2}+(x+d)^{2}\right)^{3 / 2}}\right) d \varphi_{1}+ \\
& \int_{0}^{12 \pi}\left(\frac{\left(\left(\cos \varphi_{2}-\varphi_{2} \sin \varphi_{2}\right)\left(z+(r / 12 \pi) \varphi_{2} \sin \varphi_{2}\right)\right)}{\left(\left(y+(r / 12 \pi) \varphi_{2} \cos \varphi_{2}\right)^{2}+\left(z+(r / 12 \pi) \varphi_{2} \sin \varphi_{2}\right)^{2}+(x+d)^{2}\right)^{3 / 2}}-\right. \\
& \left.\left.\frac{\left(\left(\sin \varphi_{2}+\varphi_{2} \cos \varphi_{2}\right)\left(y+(r / 12 \pi) \varphi_{2} \cos \varphi_{2}\right)\right)}{\left(\left(y+(r / 12 \pi) \varphi_{2} \cos \varphi_{2}\right)^{2}+\left(z+(r / 12 \pi) \varphi_{2} \sin \varphi_{2}\right)^{2}+(x+d)^{2}\right)^{3 / 2}}\right) d \varphi_{2}\right]
\end{aligned}
$$

where $\mu_{0}$ is the permeability of free space and $I$ is the magnitude of excitation current. Similarly, the $x$ component of the magnetic field transmitted by the $\mathrm{CC}$ is given by 


$$
B_{x}=\frac{\mu_{0} r I}{4 \pi} \int_{0}^{2 \pi} \frac{r-y \cos \varphi-z \sin \varphi}{\left((y-r \cos \varphi)^{2}+(z-r \sin \varphi)^{2}+(x+d)^{2}\right)^{3 / 2}} d \varphi
$$

\subsection{Chinese head model}

In this study, we use a human head model with real anatomical structure. This Chinese head model, provided by Third Military Medical University, is developed from the second Chinese visible human data [10]. As shown in Figure 2, the head model contains seven tissues, including scalp, skull, cerebrospinal fluid (CSF), brain, optic nerve, eyeball, and blood. The volume of the head model is about $3.8 \times 10^{6} \mathrm{~mm}^{3}$, with a resolution of $1 \mathrm{~mm}^{3}$. Proper passive electromagnetic properties can be assigned to each tissue at a specific frequency. Table 1 lists the colors and values of passive electromagnetic properties for each tissue in the head model at $10 \mathrm{MHz}$ [11]. Since muscle and white matter make up most of the scalp and brain, respectively, the passive electromagnetic properties of scalp and brain are almost equal to those of the muscle and white matter, respectively. The passive electromagnetic properties of skull and eyeball are valued using the weighted average [4]. All the tissues are assumed to be uniform, linear and isotropic mediums.
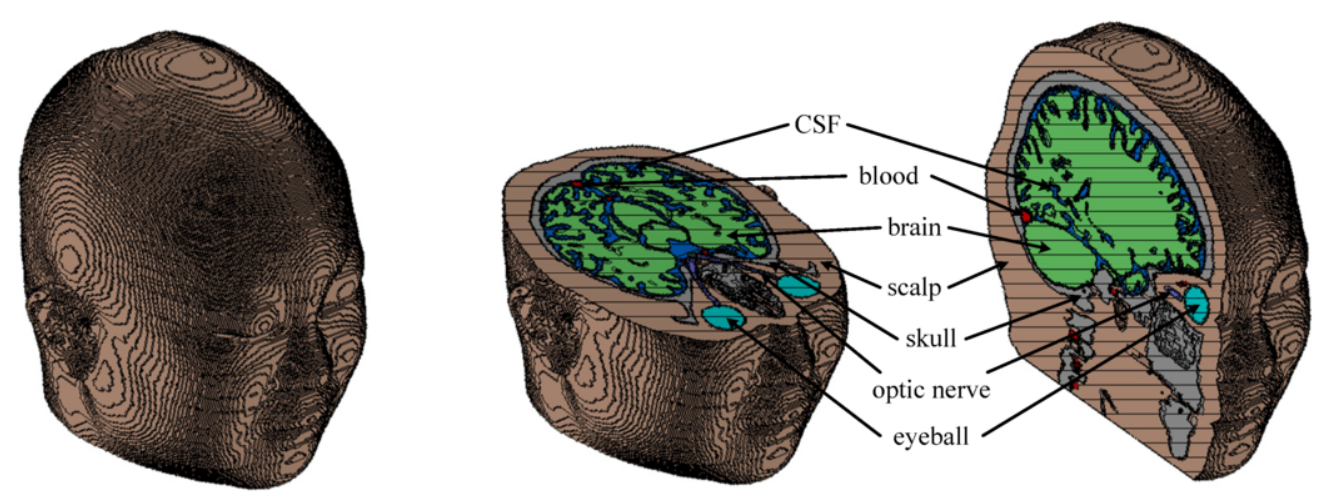

Fig. 2. Chinese head model and its cross-sectional views.

Table 1

Colors and values of passive electromagnetic properties for each tissue in the Chinese head model at $10 \mathrm{MHz}$

\begin{tabular}{|c|c|c|c|c|}
\hline Tissue & Color & Electrical Conductivity $(\mathrm{S} / \mathrm{m})$ & Relative Permittivity & Relative Permeability \\
\hline Scalp & & 0.6168 & 170.73 & 1 \\
\hline Skull & & 0.0828 & 53.78 & 1 \\
\hline CSF & & 2.0022 & 108.59 & 1 \\
\hline Brain & & 0.1585 & 175.72 & 1 \\
\hline Optic Nerve & & 0.2230 & 155.10 & 1 \\
\hline Eyeball & & 1.2000 & 127.00 & 1 \\
\hline Blood & & 1.0967 & 280.03 & 1 \\
\hline
\end{tabular}


ICH usually appears in the left or right hemisphere of the brain and above the eyeballs level. For salvageable ICH patients, the amount of bleeding in their brain ranges from 0 to $70 \mathrm{ml}$, with a moderate value of about $20 \mathrm{ml}$. When the amount is over $30 \mathrm{ml}$, it is regarded as high-volume ICH [12,13]. The passive electromagnetic properties of lesion are estimated by assuming the lesion contains 75 percent blood [4]. Therefore, the values of the electrical conductivity, relatively permittivity, and relatively permeability are $0.8622 \mathrm{~S} / \mathrm{m}, 253.95$, and 1 , respectively, referred as the average values in the paper.

\subsection{Simulation experiments}

Time-domain simulations are conducted in a three-dimensional electromagnetic simulation software (CST MW STUDIO), with generated data further processed by MATLAB (The Mathworks, Inc.). The model for simulation is presented in Figure 3. The frequency and magnitude of the excitation current are $10 \mathrm{MHz}$ and $1 \mathrm{~A}$, respectively. The coil is made of copper wire with a diameter of $1 \mathrm{~mm}$, respectively. $r$ and $d$ as defined in Section 2.2 are $50 \mathrm{~mm}$ and $110 \mathrm{~mm}$, respectively. The head model is placed in the sensor in Figure 1(b), with its ears just below the sensor to ensure that the brain is in the center of the sensor. A small hemorrhage exists in the brain at spot $(-23 \mathrm{~mm},-8 \mathrm{~mm},-22 \mathrm{~mm})$. Two simulations are carried out with details as follows.

- Simulation 1 for time sensitivity test. The hemorrhage with the average values for its passive electromagnetic properties spreads uniformly in all directions at the rate of $1 \mathrm{~mm}$ per minute. The relationship between the perturbation phase of receiver signal and the diffusion time of hemorrhage is investigated.

- Simulation 2 for state sensitivity test. The hemorrhage is treated as a sphere with a radius of 10 $\mathrm{mm}$. The passive electromagnetic properties of the hemorrhage increase linearly from those of the brain to blood. The relationship between the perturbation phase of received signal and the state for passive electromagnetic properties of hemorrhage is investigated.

In comparison, a circular excitation coil with copper material, one turn, $1 \mathrm{~mm}$ wire diameter, and 50 $\mathrm{mm}$ radius is also used for simulation.
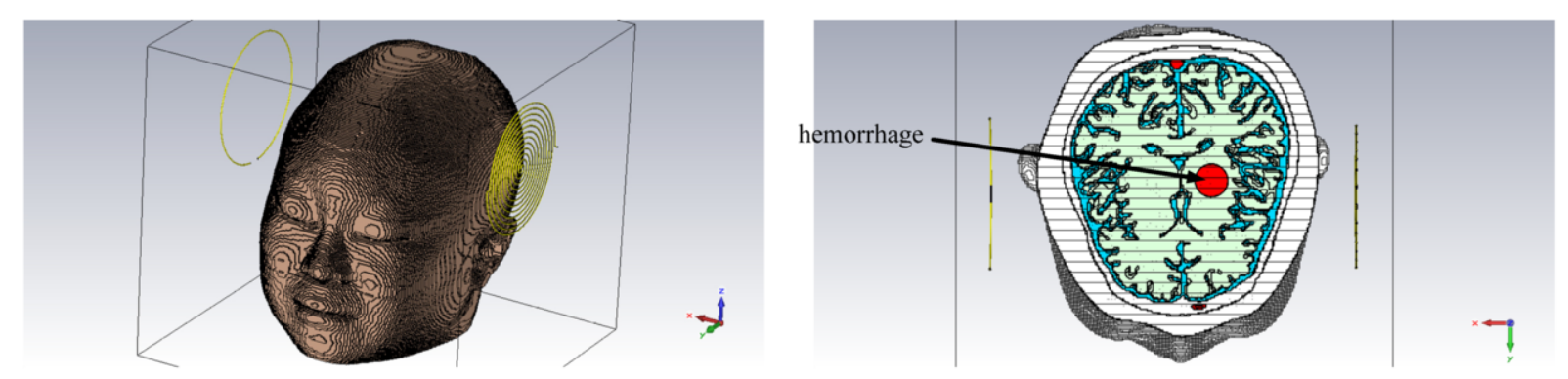

Fig. 3. Simulation model used in CST MW STUDIO. 


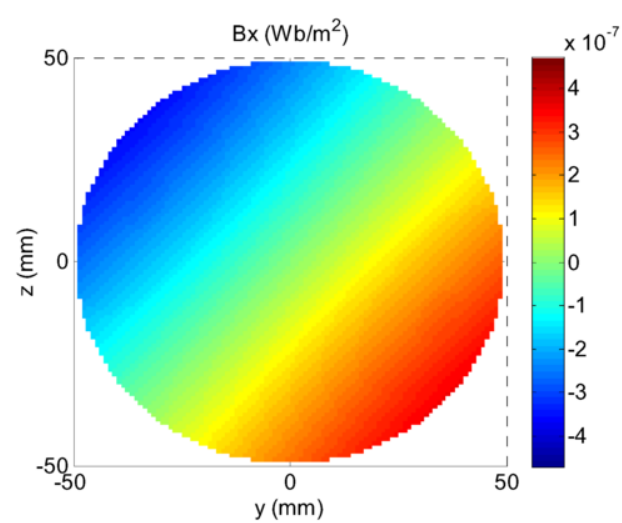

(a)

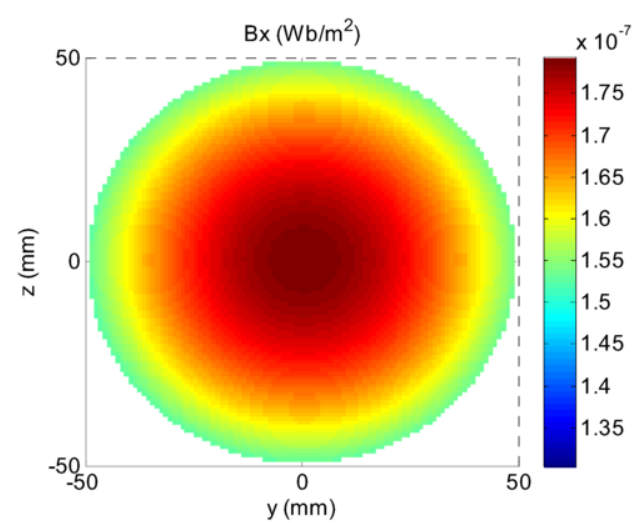

(b)

Fig. 4. Bx distribution map of the primary magnetic field at the receiver when the exciter is TAASC (a) and CC (b).

\section{Results and discussions}

\subsection{Bx distribution map of the primary magnetic field at the receiver}

Figure 4 shows the distribution maps of $x$-component of primary magnetic field at the receiver when TAASC and CC are used as the exciter, according to Eqs. (2) and (3). It can be seen that the maximum values of $B_{x}$ in both maps are almost identical, yet the distributions are quite different. In Figure 4(a) the distribution of $B_{x}$ shows a cancelling effect, where both the magnetic flux through the receiver and the induced primary voltage $V_{0}$ are quite small. According to Eq. (1), if $V_{0}$ is largely reduced, the perturbation phase $\Delta \varphi$ will be improved dramatically. Consequently, compared with CC, TAASC could effectively increase the sensitivity by reducing the primary signal.

\subsection{Perturbation phase of received signal versus diffusion time of hemorrhage}

Figure 5 shows the results of simulation 1 as described in Section 2.4. Overall, the perturbation phase tends to increase with the diffusion time. Besides, the perturbation phase when using TAASC is almost ten times as that when CC is used, and the linear fitting yields two slopes of 0.098 and 0.003 , respectively. A ratio of about 33 between these two slopes represents the average improvement factor of time sensitivity when using the novel sensor. As CST uses the finite integration method for the time-domain algorithm, it will inevitably introduce discretization errors. In addition, the computation resource is finite, and thus the mesh cannot be divided infinitesimally, which might imply large errors. As a result, some simulation data might become singular due to the discretization errors. In Figure 5(a), the simulation result at $t=6 \mathrm{~min}$ is removed as its value, 2.99 , is considerably larger than other values obtained in the simulation. Theoretically, the perturbation phase with shorter diffusion time should be lower than that with longer time. However, a perturbation object with small dimension might introduce errors easily as the mesh is coarser. Therefore, the simulation result at $t=2 \mathrm{~min}$ is slightly larger than that at $t=4 \mathrm{~min}$. 


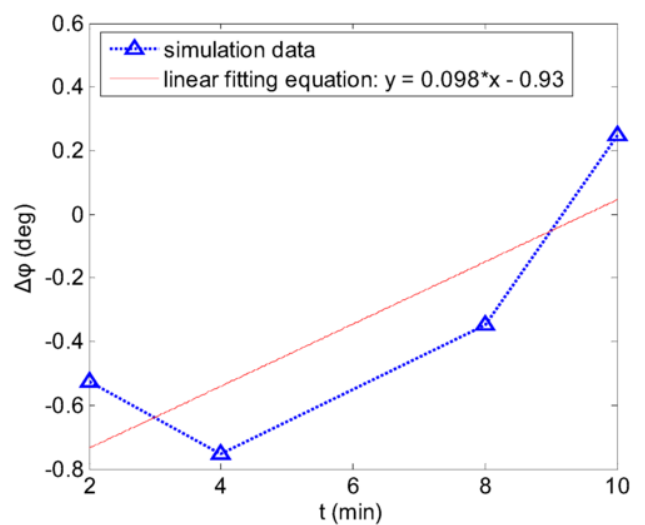

(a)

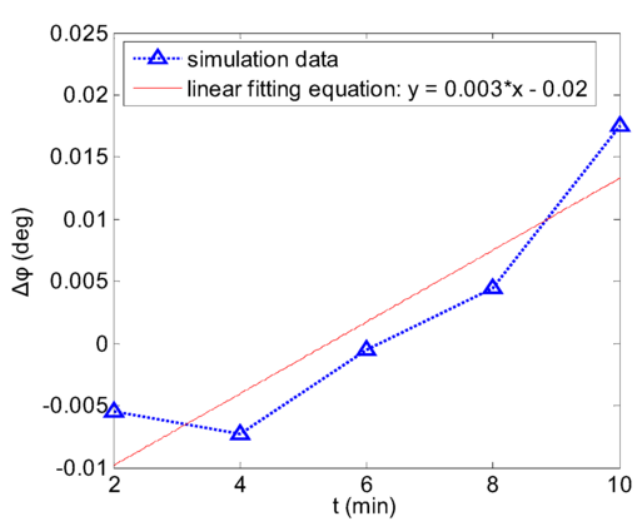

(b)

Fig. 5. Perturbation phase of received signal versus diffusion time of hemorrhage when the exciter is TAASC (a) and CC (b).

\subsection{Perturbation phase of received signal versus state for passive electromagnetic properties of hemorrhage}

Table 2 lists the values of passive electromagnetic properties of hemorrhage for different states. The term of relative permeability is absent from the table just because the values of relative permeability for the tissues in the human head are all invariable and equal to one. As shown in Figure 6, result of simulation 2 when using TAASC is almost two orders of magnitude higher than that when CC is used. As a result, it is proved that the novel sensor can improve the state sensitivity drastically. According to the simulation result, the state sensitivity is mainly affected by both parameters, i.e., the electrical conductivity and the relative permittivity. Therefore, the derivation of Eq. (1) is ideal, and the relative permittivity cannot be neglected in real situations. Due to the discretization errors from the software, the simulation result plotted in Figure 6(b) shows a strong jitter with no obvious trend. Despite this, the maximum fluctuating amplitude of the simulation result in Figure 6(b) is only about $5 \times 10^{-3} \mathrm{deg}$, which is still much smaller than that in Figure 6(a). As for reducing the discretization errors, we could either increase the mesh quantity or refine the mesh.

Table 2

Values of passive electromagnetic properties of hemorrhage for different states

\begin{tabular}{lll}
\hline State & Electrical Conductivity $(\mathrm{S} / \mathrm{m})$ & Relative Permittivity \\
\hline 1 & 0.34614 & 196.582 \\
2 & 0.53378 & 217.444 \\
3 & 0.72142 & 238.306 \\
4 & 0.90906 & 259.168 \\
5 & 1.09670 & 280.030 \\
\hline
\end{tabular}




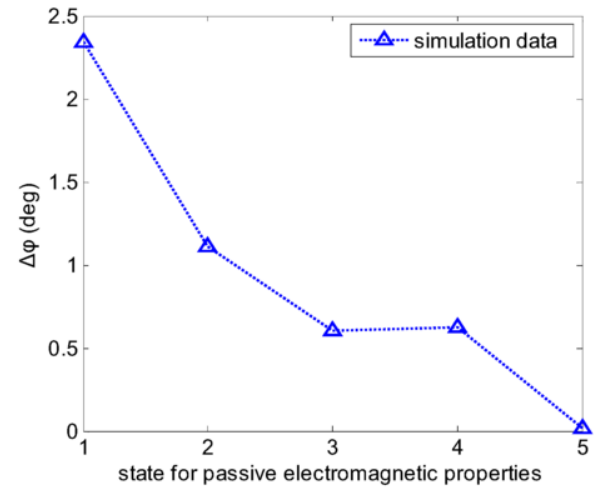

(a)

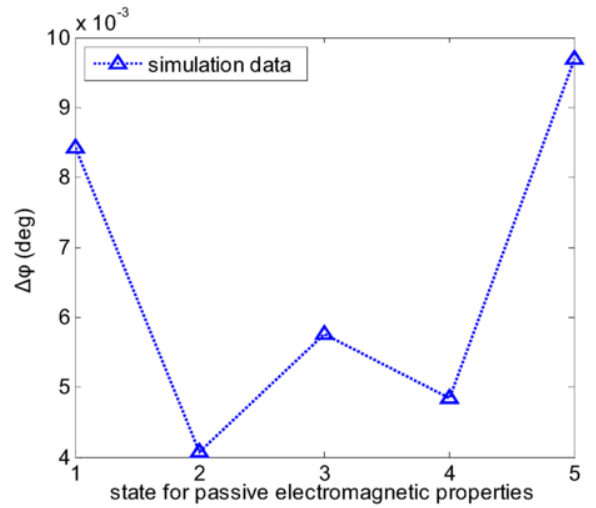

(b)

Fig. 6. Perturbation phase of received signal versus state for passive electromagnetic properties of hemorrhage when the exciter is TAASC (a) and CC (b).

\section{Conclusion}

In this paper, we investigate the sensitivity of the novel magnetic induction sensor, and conduct simulations to measure early stage ICH. The object under investigation is the Chinese head model with real anatomical structure. As there exist differences in the size, anatomical structure and passive electromagnetic properties of eastern and western people's heads, it is necessary to choose an appropriate head model to conduct simulations for ICH measurements. This paper reports ICH studies on eastern people's head model based on the magnetic induction method for the first time. The head model used comes from the Chinese, which represents the people from the eastern world. The simulation results show that, compared with conventional sensors used, a novel sensor can improve the time sensitivity and state sensitivity by one and two orders of magnitude, respectively. As a result, this method could be applied in early stage ICH measurements. This paper presents preliminary research, and future work would include more experiments to confirm the applicability of this method.

\section{Acknowledgment}

This work was supported by the National Natural Science Foundation (No. 61372029) from the People's Republic of China, the Specialized Research Fund for the Doctoral Program of Higher Education (No. 20114307110022) from Ministry of Education of the People's Republic of China, and the Innovation Fund for Doctoral Candidate (No. B130205) from National University of Defense Technology. The authors would like to thank the Associate Professor X. Ning from Third Military Medical University and the Engineer H. Li from No. 161 Hospital of PLA for helpful discussions, and are also grateful to Third Military Medical University for providing the Chinese head model.

\section{References}

[1] C. Tsai, B. Thomas and C. Sudlow, Epidemiology of stroke and its subtypes in Chinese vs white populations: a systematic review, Neurology 81 (2013), 264-272. 
[2] L. Liu, D. Wang, K.S. Wong and Y. Wang, Stroke and stroke care in China: huge burden, significant workload, and a national priority, Stroke 42 (2011), 3651-3654.

[3] H. Scharfetter, R. Casañas and J. Rosell, Biological tissue characterization by magnetic induction spectroscopy (MIS): requirements and limitations, IEEE Transactions on Biological Engineering 50 (2003), 870-880.

[4] M. Zolgharni, P. Ledger and H. Griffiths, Forward modelling of magnetic induction tomography: a sensitivity study for detecting haemorrhagic cerebral stroke, Medical \& Biological Engineering \& Computing 47 (2009), 1301-1313.

[5] Y. Chen, M. Yan, D. Chen, M. Hamsch, H. Liu, H. Jin, M. Vauhkonen, C. Igney, J. Kahlert and Y. Wang, Imaging hemorrhagic stroke with magnetic induction tomography: realistic simulation and evaluation, Physiological Measurement 31 (2010), 809-827.

[6] J. Sun, G. Jin, M. Qin, Z. Wan, J. Wang, C. Wang, W. Guo, L. Xu, X. Ning, J. Xu, X. Pu, M. Chen and H. Zhao, The detection of chronic cerebral hemorrhage in rabbits with magnetic induction, Journal of Physics: Conference Series 407 (2012), 012014.

[7] B. Dekdouk, Image reconstruction of low conductivity material distribution using magnetic induction tomography, Ph.D. Dissertation, University of Manchester, 2010.

[8] H. Wei and M. Soleimani, Electromagnetic tomography for medical and industrial applications: challenges and opportunities, Proceedings of the IEEE 101 (2013), 27-46.

[9] H. Griffiths, Magnetic induction tomography, Measurement Science and Technology 12 (2001), 1126-1131.

[10] S. Zhang, P. Heng, Z. Liu, L. Tan, M. Qiu, Q. Li, R. Liao, K. Li, G. Cui, Y. Guo, X. Yang, G. Liu, J. Shan, J. Liu, W. Zhang, X. Chen, J. Chen, J. Wang, W. Chen, M. Lu, J. You, X. Pang, H. Xiao and Y. Xie, Creation of the Chinese visible human data set, The Anatomical Record Part B: The New Anatomist 275B (2003), 190-195.

[11] S. Gabriel, R. Lau and C. Gabriel, The dielectric properties of biological tissues: III. Parametric models for the dielectric spectrum of tissues, Physics in Medicine and Biology 41 (1996), 2271-2293.

[12] M. Wasay, A. Yousuf, D. Lal and S. Awan, Predictors of the intracerebral hemorrhage volume in hypertensive patients, Cerebrovascular Diseases Extra 1 (2011), 1-5.

[13] F. Magistris, S. Bazak and J. Martin, Intracerebral hemorrhage: pathophysiology, diagnosis and management, McMaster University Medical Journal 10 (2013), 15-22. 